\title{
Exploiter la vidéo dans les dispositifs de formation des enseignants novices : conceptualisation et discussion théoriques à partir d'une étude de cas en EPS
}

Using video in pre-service teacher education: theoretical conceptualization and discussion from a case study in physical education

Sébastien Chaliès, Cyrille Gaudin et Hervé Tribet

\section{(2) OpenEdition}

Journals

Édition électronique

URL : http://journals.openedition.org/rfp/4880

DOI : $10.4000 /$ rfp.4880

ISSN : 2105-2913

Éditeur

ENS Éditions

Édition imprimée

Date de publication : 31 décembre 2015

Pagination : 5-24

ISBN : 978-2-84788-863-8

ISSN : 0556-7807

Référence électronique

Sébastien Chaliès, Cyrille Gaudin et Hervé Tribet, «Exploiter la vidéo dans les dispositifs de formation des enseignants novices : conceptualisation et discussion théoriques à partir d'une étude de cas en EPS », Revue française de pédagogie [En ligne], 193 | octobre-novembre-décembre 2015, mis en ligne le 31 décembre 2018, consulté le 03 janvier 2020. URL : http://journals.openedition.org/rfp/4880 ; DOI : $10.4000 /$ rfp.4880 


\title{
Exploiter la vidéo dans les dispositifs de formation des enseignants novices : conceptualisation et discussion théoriques à partir d'une étude de cas en EPS
}

\author{
Sébastien Chaliès \\ Cyrille Gaudin \\ Hervé Tribet
}

\begin{abstract}
Cet article présente les principaux postulats d'une théorie singulière permettant de réinterroger la principale controverse scientifique subsistant actuellement dans la littérature relative à l'utilisation de la vidéo dans les dispositifs de formation professionnelle des enseignants novices. Cette théorie emprunte ses postulats à un programme de recherche mené en anthropologie «culturaliste » et fondé sur la philosophie analytique de Wittgenstein. Après avoir été détaillée, cette théorie est ensuite illustrée à partir de résultats empiriques extraits d'une étude de cas menée en EPS. Une discussion à dimension théorique est finalement proposée pour réinterroger les modalités «normative» et « développementale» d'utilisation de la vidéo en formation et alimenter une conceptualisation de la construction des sujets professionnels.
\end{abstract}

Mots-clés (TESE) : formation des enseignants, théorie de l'enseignement, équipement audiovisuel, éducation physique.

\section{Introduction}

Au sein de la littérature, un consensus existe quant aux intérêts de la vidéo pour optimiser la formation des enseignants novices (EN). Une lecture approfondie de cette littérature permet néanmoins de situer la principale controverse scientifique qui subsiste. Celle-ci est relative aux modalités d'utilisation de la vidéo au sein des dispositifs de formation proposés aux EN (Gaudin \& Chaliès, 2012; Gaudin, Flandin, Ria et al., 2014). 
Étayées par des cadres théoriques voire des ancrages épistémologiques différents' ${ }^{1}$, deux grandes modalités apparaissent en matière d'usage de la vidéo en formation (Gaudin \& Chaliès, 2015).

Dans la première et principale modalité, la vidéo est utilisée dans le cadre de dispositifs pensés selon une approche dite «développementale». L'objectif poursuivi est alors celui de la construction du «comment interpréter et réfléchir aux pratiques de classe» (Sherin, 2004, p.14). La vidéo est exploitée pour confronter les EN à une multiplicité de pratiques professionnelles et stimuler leur réflexion (Brophy, 2004). L'objectif est de leur présenter sous la forme de vidéos choisies des "exemples non exemplaires» (Santagata \& Guarino, 2011) pouvant servir de support pour l'analyse et la discussion sur l'enseignement et l'apprentissage. Parmi les principaux dispositifs, ceux de type «apprentissage par résolution de problèmes» (Syring, Kleinknecht, Bohl et al., 2015) ou "analyse de leçon» (Santagata \& Yeh, 2014) occupent une place significative. Des dispositifs de type «microenseignement» (Ostrosky, Mouzourou, Danner et al., 2013) ou «jeux de rôle» (Koc, 2011) sont aussi mis en œuvre pour construire à partir de vidéos le «comment interpréter et réfléchir» chez des EN. D'autres dispositifs de type "clubs vidéo» ont aussi été élaborés pour construire la capacité des EN à identifier et interpréter certains événements dans la classe (Sherin \& van Es, 2005). Enfin, des dispositifs permettant une "analyse d'incidents critiques» réels (Calandra \& Brantley-Dias, 2010) ou virtuels (Puvirajah \& Calandra, 2015) sont aussi exploités dans de nombreuses études.

Dans la seconde modalité, la vidéo est utilisée dans le cadre de dispositifs de formation pensés selon une approche dite «normative». L'objectif poursuivi est alors celui de la construction du «que faire» en classe (Brouwer, 2011). L'intérêt premier de l'utilisation de la vidéo se situe alors dans les possibilités qu'elle offre en matière de présentation de pratiques professionnelles pouvant être considérées comme «exemplaires » dans les circonstances d'enseignement considérées (Merseth, 1994). Sélectionnées puis diffusées en ce sens, les vidéos sont alors assimilées à des exemples de « bonnes pratiques » d'enseignement (Marsh, Mitchell \& AdamCzyk, 2009) ou à des exemples de leçons typiques (Yung, Wong, Cheng et al., 2007).

1 Pour une analyse historique et contemporaine des différents cadres théoriques mobilisés dans les études portant sur la vidéoformation, voir Flandin, 2015a.
Même si leurs ancrages théoriques semblent difficilement conciliables, quelques rares études ont tenté d'articuler ces deux modalités (développementale et normative) d'utilisation de la vidéo au sein d'un même dispositif de formation (par exemple : Masats \& Dooly, 2011).

De nature théorique, cet article a pour principale finalité d'aider au dépassement de cette controverse scientifique considérée comme la plus significative dans la littérature scientifique du domaine. Pour ce faire, il est structuré pour 1) permettre la présentation d'une conceptualisation théorique originale de la formation à partir de laquelle cette controverse peut être interrogée de façon heuristique, 2) illustrer cette conceptualisation à partir des résultats d'une étude empirique menée sur un dispositif de formation des EN en EPS exploitant la vidéo et 3) discuter des contributions envisageables de cette conceptualisation en matière de réponse à la controverse scientifique préalablement délimitée. Lors de cette discussion, des conceptualisations théoriques proximales seront mobilisées afin de tracer finalement, en conclusion, de nouveaux axes de travaux scientifiques.

\section{Cadre théorique}

Sur la base de la classification proposée récemment par Flandin (2015b), le cadre théorique adopté relève d'une approche de la vidéoformation «centrée activité». À ce titre, il peut être considéré sur un grand nombre de ses soubassements comme proche d'autres cadres récents mobilisés dans le paysage francophone pour étudier les modalités d'utilisation de la vidéo au sein des dispositifs de formation et apprécier leurs effets sur le développement professionnel des EN. Sans chercher à être exhaustif, c'est le cas par exemple des travaux récents menés en anthropologie dite cognitive mobilisant la théorie sémiologique du cours d'action de Theureau (2004, 2006; Flandin \& Ria, 2014; Roche \& Gal-Petitfaux 2015) tout en cherchant à l'enrichir de concepts empruntés à d'autres théories comme celui de la «renormalisation » emprunté à Schwartz (2007; Lussi Borer \& Muller, 2014a, 2014b), celui d'«enquête» emprunté à Dewey (1990; Lussi Borer \& Ria, 2015; Lussi Borer, Ria, Durand et al., 2014), ou encore celui d'«invention pratique» emprunté à Simondon (2008; Leblanc, 2012, 2014). D'autres travaux (Ciavaldini-Cartaut, 2016; Moussay, 2013) étudient les situations de vidéoformation à partir d'une approche historico-culturelle (Vygotski, 1997) et 
de la clinique de l'activité (Clot, 2008). Le cadre théorique de cet article emprunte ses principaux postulats à un programme de recherche mené en anthropologie «culturaliste» (Bertone, Chaliès \& Clot, 2009; Chaliès, Amathieu \& Bertone, 2013) principalement inspiré de la philosophie analytique de Wittgenstein (2004). Essentiels compte tenu de l'objet de l'article, deux de ces postulats sont ci-après explicités.

\section{Travailler et se former au travail nécessite de s'engager dans un double régime de réflexivité}

Au sein de ce cadre théorique, travailler et/ou se former au travail revient à mener des actions «gouvernées par des règles » et/ou à réaliser des actions qui sont «le suivi de règles" (Ogien, 2007). Ces règles (Wittgenstein, 2004) sont des "expériences situées normatives» (Lähteenmäki, 2003) acceptées par la communauté professionnelle, rendant intelligibles, anticipables et évaluables les actions de chacun (Livet, 1993). Ces règles ont une autorité sur les membres de la communauté car elles sont des standards de correction acceptés dans la communauté professionnelle considérée. Cependant, elles ne déterminent pas leur propre application en ce que l'acteur qui les suit peut à tout instant s'en écarter, décider de les transgresser ou en rejeter le suivi (Descombes, 2004).

Lorsqu'il travaille ou se forme à son travail, chaque acteur s'engage dans différents registres de réflexivité par rapport à ces règles (Grammont, Legrand \& Livet, 2011). Dans certaines circonstances de travail ou de formation, l'acteur est engagé dans un régime de réflexivité de l'ordre de "l'inhérence» (Searle, 1998) relevant d'actions et de significations gouvernées par des règles apprises au travail ou en formation. Il agit selon ces dernières qui, à l'instant considéré, ne lui sont pas conscientes ni par-là même dicibles. Qu'il soit en formation ou au travail, l'acteur réalise en effet un certain nombre d'actions dont l'apprentissage s'est fait implicitement par l'intermédiaire d'interactions non verbales et/ou d'alignements informels avec la pratique des autres acteurs au cours de sa participation aux pratiques professionnelles constitutives de la communauté (Winch, 2009). Dans d'autres circonstances de travail ou de formation, l'acteur est par contre engagé dans un régime de réflexivité de l'ordre de «l'appréhension de l'inhérence » (Searle, 1998) relevant des capacités pratiques, apprises en formation, à invoquer telle ou telle règle suivie pour justifier ce que l'on fait, a fait ou voudrait faire. II réalise alors des actions qui sont le «suivi de règles» (Ogien, 2007). Il agit donc en conformité avec ces dernières et peut donc s'appuyer sur elles pour se justifier. Elles lui sont en effet conscientes et dicibles parce qu'elles ont été apprises lors d'une formation antérieure au cours de laquelle il a appris avec l'aide d'un ou plusieurs formateurs à en prendre conscience, c'est-à-dire à les rendre intelligibles par l'intermédiaire de réélaborations discursives (Legrand, 2007).

Concernant la formation des EN, le principe de l'alternance entre les séquences de formation au travail à l'université et/ou en établissement scolaire et les séquences de travail en classe, constitutif des dispositifs exploitant la vidéo, est principalement fondé à partir de ce second régime de réflexivité. L'idée n'est toutefois pas ici de considérer que le régime de réflexivité de l'ordre de «l'inhérence » n'est pas mis en jeu lors de ce type d'alternance. Certains auteurs ont d'ailleurs montré, en s'appuyant sur d'autres cadres conceptuels, que des apprentissages implicites se font par mimétisme lors du visionnage d'extraits vidéo de pairs (Flandin, Leblanc \& Muller, 2015). Dans le cadre de I'anthropologie culturaliste, ce résultat a aussi été obtenu. C'est par exemple ce qui a été mis en évidence par l'étude des effets formatifs sur des EN de dispositifs de formation intégrant des stages d'observation de la pratique d'enseignement de leurs tuteurs (Chaliès, Bertone, Flavier et al., 2008). Dans ce type de situations de formation, les EN se saisissent en effet régulièrement, à l'insu des tuteurs, de faits « tacites » et pourtant observables au sein de leur enseignement. Autrement dit, les EN bénéficient de l'engagement des tuteurs dans un enseignement ostensible de nouveaux faits aux EN sans forcément en avoir pleinement conscience (Burbules, 2008). Néanmoins, le principe de l'alternance reste encore de nos jours largement fondé sur le primat donné à une "conception réflexive» de la formation (Tardif, Borges \& Malo, 2013). C'est à ce titre que le régime de «l'appréhension de l'inhérence » est explicité ci-dessous.

\section{Une formation professionnelle par alternance : faire apprendre les règles aux enseignants novices puis leur permettre de les interpréter}

L'engagement des EN dans une activité d'enseignement en classe ou de formation de type analyse de pratique professionnelle à l'université via un support 
vidéo nécessite un apprentissage préalable de règles. Ces dernières leur permettent en effet respectivement soit d'adapter leurs actions aux circonstances de classe soit de signifier, d'analyser voire de proposer des remédiations aux difficultés visionnées puis abordées en formation. Cet apprentissage nécessite l'engagement des formateurs dans une activité d'«enseignement ostensif» (Wittgenstein, 2004) par laquelle ils fondent comme «exemplaires» (Laugier, 2009) certaines expériences professionnelles, autrement dit certaines règles. Pour ce faire, ils dressent pour chacune de celles-ci un «lien de signification» (Bertone, Chaliès \& Clot, 2009) entre 1) une expérience langagière visant à la nommer, 2) un exemple exemplaire décrit et/ou donné à observer en correspondance et 3 ) des résultats décrits et/ou donnés à observer obtenus dans le cas d'un suivi conforme de la règle considérée. Ainsi, par exemple, le formateur peut enseigner à ses étudiants ce qu'est "présenter une situation d'apprentissage». Pour ce faire, il nomme cette règle et lui associe en le commentant verbalement un extrait vidéo de pratique professionnelle jugée par lui comme exemplaire et contenant un enseignant qui «stoppe le travail de ses élèves, les rassemble, leur délivre de façon ordonnée des consignes, juge de leur compréhension et les relance en activité». Ensuite, il précise verbalement aux EN que, suivie de façon conforme, cette règle doit permettre d'obtenir «une compréhension et une exploitation rapides des consignes par les élèves $»^{2}$.

Suite à leur enseignement ostensif par les formateurs, les règles deviennent alors potentiellement pour les EN de véritables expériences «mètre étalon" (Williams, 2002). Autrement dit, les EN peuvent alors s'essayer à observer, décrire et/ou agir comme ceci ou cela, c'est-à-dire en tant que ceci ou cela, parce qu'ils sont en capacité de dire et de faire ce qui «vaut pour » ceci ou cela. Observer et/ou agir en situation de formation ou de classe revient donc à faire preuve d'un discernement, c'est-à-dire à observer et/ou agir sur la base d'une grammaire expérientielle, faite de règles, enseignée en amont (Laugier \& Chauviré, 2006). Toutefois, ces expériences "mètre étalon » enseignées ne sont pas à considérer comme une "normalité étalon » (Le Blanc, 2004) que les EN se devraient d'appliquer. Elles sont à assimiler à un exemple canonique initialement exemplaire (Chauviré, 2010) à partir duquel ils vont pouvoir ensuite reconnaître en situation de classe

2 Cet exemple de règle est emprunté à Gaudin, Perrot, Chaliès et al. (2014). ou de formation des circonstances présentant un «air de famille» (Wittgenstein, 2004) et agir en conséquence. Les formateurs ne peuvent donc pas se suffire d'une activité d'enseignement ostensif des règles pour que celles-ci soient apprises par les EN. Ils doivent ensuite leur proposer d'en user, c'est-à-dire de s'engager dans leurs «premiers suivis» (Berducci, 2004) au sein de situations de formation, dont certaines peuvent exploiter la vidéo, puis de situations de classe. Par cette activité, ils cherchent à aménager le contexte de ces situations de formation ou de classe afin d'autoriser les EN à suivre les règles préalablement enseignées, c'està-dire à se retrouver dans des circonstances leur permettant de "voir comme», par exemple en situation d'analyse de pratiques visionnées, mais aussi et surtout d'« agir comme» cela leur a été antérieurement enseigné lorsqu'ils sont en classe.

Les formateurs cherchent plus précisément à faire en sorte que les premiers suivis des règles par les EN aboutissent aux résultats attendus leur étant usuellement associés. Ce n'est en effet qu'au travers du constat de ces résultats que ces derniers pourront alors rattacher une intention professionnelle concrète à la règle enseignée (Cash, 2009). C'est donc en suivant correctement les règles préalablement enseignées et en constatant en classe les résultats qui leur sont usuellement associés dans la communauté que les EN finaliseront leurs apprentissages et pourront alors se développer professionnellement et subjectivement en leur sein (Nelson, 2008). Lors de ces premiers suivis, les formateurs en "contrôlent» la conformité au regard des règles enseignées et s'engagent, si nécessaire, dans une activité d'«explication ostensive» (Davis, 2009). Ils s'efforcent alors de multiplier les exemples décrits, donnés à visionner et/ou démontrés, de sorte que les EN se saisissent de l'exemplaire contenu par essence, comme un «noyau de ressemblances» (Laugier, 2009), dans chacun des exemples et finalement sortent de leurs éventuelles mésinterprétations.

Lorsque l'apprentissage des règles est effectif, les EN sont alors en mesure de s'émanciper du contrôle des formateurs. Progressivement, ils parviennent à construire un système d'interprétations des règles (Winch, 2009) qui leur autorise un usage «extensif » des liens de signification construits et appris au-delà des situations d'apprentissage originelles. C'est sur la base d'une identification d'un "air de famille» entre les circonstances de la situation en cours et celles constitutives des situations originelles de formation que cet usage extensif est possible. À partir des liens de signi- 
fication appris, les EN ordonnent finalement un réseau complexe de ressemblances, "comprennent» la nouvelle situation en cours, et, in fine, parviennent à produire des activités conformes aux attentes des membres de la communauté, tout en s'émancipant progressivement de leur contrôle. Lors de ces situations de développement, les formateurs ne cherchent plus à contrôler le caractère adéquat ou inadéquat de I'activité des EN. Ils s'efforcent de les aider à s'engager dans des suivis singuliers des règles apprises, c'est-àdire de les aider à en négocier la signification originelle pour mieux en user dans des circonstances toujours singulières de travail. Plus largement, la distinction établie en amont entre l'apprentissage des règles et le développement professionnel qu'elles ouvrent potentiellement rapproche les soubassements théoriques de l'anthropologie culturaliste de ceux de la psychologie historico-culturelle déployés en psychologie du travail (voir pour plus de détails sur cette tentative de rapprochement: Bertone, Chaliès \& Clot, 2009). Sans basculer dans une lecture psychologique, il semble effectivement possible de soutenir que l'apprentissage des règles par les EN ouvre la voie à une «zone de développement potentiel» (Clot, 2008) de leur subjectivité. En faisant ce qu'ils ont appris à faire dans de nouvelles circonstances, autrement dit dans des situations différentes de celles lors desquelles l'enseignement ostensif des règles et leurs premiers suivis ont été antérieurement réalisés, les EN construisent leur subjectivité, c'est-à-dire se développent. Autrement dit, toute activité de développement des EN ne coïncide donc pas avec celle de leur apprentissage des règles constitutives du genre professionnel (Clot, 2008) mais par contre y fait nécessairement suite. Finalement, «le seul bon enseignement est [donc] celui qui précède le développement»(Vygotski, 1985, p. 109).

\section{Question de recherche}

Compte tenu des postulats théoriques exposés en amont, il semble possible de réinterroger la principale controverse subsistant dans la littérature du domaine relative aux modalités d'utilisation de la vidéo au sein des dispositifs de formation proposés aux EN. Une question de recherche peut être en ce sens formalisée: afin que ses retombées sur la formation des EN soient effectives, I'utilisation de la vidéo ne devrait-elle pas être pensée à partir d'une articulation théoriquement fondée des approches «normative» et «développementaliste » tout au long des dispositifs de formation?

\section{Méthode}

Pour illustrer le cadre théorique développé en amont et discuter de ses potentielles contributions en matière de réponse à la controverse scientifique préalablement délimitée, nous nous appuyons sur les résultats d'une étude de cas dont les principales caractéristiques sont les suivantes.

\section{Participants}

Cette étude a été réalisée avec une triade composée d'un EN, d'un formateur de terrain (FT) et d'un formateur de l'université (FU). Âgé de 24 ans, l'EN était un étudiant de Master 2 Métiers de l'enseignement, de l'éducation et de la formation (MEEF) se destinant au professorat d'EPS. Au moment de l'étude, son expérience d'enseignement se limitait à deux stages d'observation de deux semaines et un stage de pratique accompagnée avec un enseignant titulaire, soit un total de 24 journées en établissement scolaire. Âgé de 36 ans, le FT était à la fois considéré comme un enseignant mais aussi comme un tuteur expérimenté (respectivement 11 et 7 années d'expérience dans chacune de ces fonctions). Âgé de 38 ans, le FU avait une expérience d'enseignant en établissement scolaire de 7 années. Il était engagé dans la formation des enseignants novices depuis 8 années.

\section{Dispositif}

Le dispositif de formation support à l'étude était celui d'une des sept unités d'enseignement (UE) constitutives du Master 2 MEEF. D'une durée de 24 heures, cette UE avait été sélectionnée pour deux raisons. D'une part, elle était la seule simultanément encadrée par un FT et un FU. Le principal objectif des deux formateurs était de délivrer aux EN des contenus relatifs à trois règles étiquetées : «préparer et mettre en œuvre son enseignement»; « «aire apprendre les élèves » et « différencier son enseignement $»^{3}$.

3 D'un point de vue professionnel, le collectif de formateurs regroupant des FT et des FU s'est appuyé sur les propositions conceptuelles et méthodologiques de la clinique de l'activité (Clot, 1999) pour formaliser les règles de métier constitutives du «genre professionnel», objet partagé de la formation avec les EN. Pour plus de renseignements sur ce point, se référer aux productions «professionnelles » suivantes: Chaliès \& Raymond, 2008; Recoules \& Chaliès, 2011 ; Recoules, Escalié, Gaudin et al., 2012; Gaudin, Perrot, Chaliès et al., 2014. 
D’autre part, cette UE était organisée sous la forme d'une alternance entre des séquences de formation par le visionnage d'extraits vidéo réalisés à l'université avec les formateurs ou de la pratique professionnelle du FT auprès de ses élèves en classe et des séquences d'enseignement des EN en classe. Précédées d'une séquence de préparation du dispositif, les cinq séquences constitutives de I'UE étaient ordonnées comme suit :

- séquence de préparation du dispositif de formation regroupant le FU en charge de celui-ci et les FT volontaires. Lors de cette séquence, le FU nomme les différentes règles qui seront l'objet de la formation des EN. II présente ensuite les exemples exemplaires et les résultats attendus qui avaient été associés à ces règles lors des années antérieures. Une discussion est ensuite engagée jusqu'à ce qu'un accord soit obtenu quant à la formalisation de chaque règle. Pour chacune d'entre elles, un étiquetage, un exemple exemplaire et des résultats attendus sont donc arrêtés par le collectif de formateurs. Parmi les FT, un volontaire s'engage à recevoir les EN lors d'une de ses leçons et à mettre en œuvre les règles préalablement formalisées. Cette mise en œuvre est enregistrée; - séquence 1 (S1) : formation des EN par le FT en classe par l'observation de son activité d'enseignement;

- séquence 2 (S2) : formation des EN par les deux formateurs (FU et FT) à l'université par le visionnage d'extraits vidéo enregistrés lors de la leçon du FT (S1). Ces extraits sont sélectionnés par les deux formateurs sur la base de leur caractère exemplaire de la règle objet de formation. Théoriquement, ils servent aux formateurs à exemplariser la règle lors de son enseignement ostensif ou, par leur multiplicité, à appuyer les explications ostensives;

- séquence 3 (S3) : pratique d'enseignement des EN auprès des élèves de la classe du FT observée en $\mathrm{S1}$;

- séquence 4 (S4) : formation des EN par les deux formateurs (FU et FT) à l'université par le visionnage d'extraits vidéo enregistrés lors de la leçon d'autres FT (années antérieures). Ces extraits sont sélectionnés par le FU sur la base de leurs différences plus ou moins significatives avec l'exemple de la règle initialement enseignée aux EN. Théoriquement, ils servent aux formateurs à engager les EN dans une interprétation de la règle préalablement apprise; - séquence 5 (S5) : pratique d'enseignement des EN auprès d'élèves différents de ceux de la S3.

\section{Recueil des données}

Deux catégories de données ont été recueillies pour composant le dispositif, des données d'enregistrement audio-vidéo ont d'abord été recueillies. Une caméra vidéo et un micro HF ont été utilisés pour ce recueil. Outre ce recueil de données d'enregistrement extrinsèques, le recueil des données a aussi été réalisé par la confrontation des acteurs aux traces de leurs propres expériences. Pour y parvenir, le chercheur a procédé par I'intermédiaire d'entretiens d'autoconfrontation (EAC). Ces données intrinsèques dites d'autoconfrontation ont aussi été recueillies au cours d'enregistrements audiovidéo. Pour chaque séquence, chacun des acteurs présents (EN, FU et/ou $\mathrm{FT}$ ) a réalisé avec le chercheur un EAC. Au total, 11 entretiens, d'une durée de 45 minutes à une heure, ont été réalisés (voir tableau 1).

Lors de ces EAC, l'objectif du chercheur était la remise en situation des acteurs en provoquant les conditions d'une immersion mimétique dans la situation d'enseignement, de travail et/ou de formation observée (Durand, 2008). L'enjeu était pour le chercheur de demander à l'acteur autoconfronté de suspendre toute analyse de son expérience. Plus précisément, le chercheur a accédé aux critères d'intelligibilité de l'action de l'acteur «en se faisant instruire» par lui sur la signification de ses actions et ce par l'engagement d'un questionnement permettant de soutenir une sorte d'« étayage à l'envers» (Ogien, 2007) lui permettant d'apprendre à signifier comme lui et à faire comme si c'était à lui d'agir conformément aux modes opératoires énoncés. Dans le détail, les EAC ont été conduits de façon à pouvoir reconstituer a posteriori les règles suivies par les acteurs au cours des séquences de formation et/ou de classe. Par un questionnement structuré à partir du cadre conceptuel adopté (pour plus de détails sur cette construction, voir Gaudin \& Chaliès, 2015), le chercheur incitait l'acteur interviewé à l'instruire sur les significations à attribuer aux actions observées et aux jugements pouvant y être associés («Qu'est-ce que te propose le formateur à ce moment-là?»). En lui demandant des précisions (« Je ne comprends pas pourquoi tu le questionnes, peux-tu m'en donner les raisons? ») ou en jouant sur la controverse en plaçant l'acteur interviewé face à des contradictions apparentes («Tu cherches à stopper la vidéo mais apparemment tu tâtonnes encore. Tu viens pourtant de lui dire que tu avais compris non?»), le chercheur invitait ensuite l'acteur à étayer les jugements préalablement portés sur les actions signifiées. Enfin, il invitait l'acteur à préciser les résultats attendus quant aux actions observées («Qu'est-ce que tu cherches lorsque tu lui poses cette question?»). 
Tableau 1. Séquences, types de situation de formation et acteurs impliqués

\begin{tabular}{|c|c|c|c|c|c|}
\hline Séquences & Séquence 1 & Séquence 2 & Séquence 3 & Séquence 4 & Séquence 5 \\
\hline Lieu de formation & $\begin{array}{l}\text { Établissement } \\
\text { scolaire }\end{array}$ & $\begin{array}{l}\text { Centre de } \\
\text { formation }\end{array}$ & $\begin{array}{l}\text { Établissement } \\
\text { scolaire }\end{array}$ & $\begin{array}{l}\text { Centre de } \\
\text { formation }\end{array}$ & $\begin{array}{l}\text { Établissement } \\
\text { scolaire }\end{array}$ \\
\hline $\begin{array}{l}\text { Type de situation } \\
\text { de formation }\end{array}$ & $\begin{array}{l}\text { Observation } \\
\text { par les EN de la } \\
\text { pratique de } \\
\text { classe du FT }\end{array}$ & $\begin{array}{l}\text { Formation des } \\
\text { EN par le FT } \\
\text { et le FU }\end{array}$ & $\begin{array}{l}\text { Enseignement } \\
\text { des EN dans la } \\
\text { classe du FT }\end{array}$ & $\begin{array}{l}\text { Formation des } \\
\text { EN par le FT } \\
\text { et le FU }\end{array}$ & $\begin{array}{l}\text { Enseignement } \\
\text { des EN dans un } \\
\text { autre contexte } \\
\text { classe }\end{array}$ \\
\hline \multicolumn{6}{|l|}{ Acteurs impliqués } \\
\hline EN & $P(E A C)$ & $P(E A C)$ & $P(E A C)$ & $P(E A C)$ & $P(E A C)$ \\
\hline FT & $P(E A C)$ & $P(E A C)$ & $P(E A C)$ & $P(E A C)$ & A \\
\hline FU & $A$ & $P(E A C)$ & A & $P(E A C)$ & A \\
\hline
\end{tabular}

Légende : P : acteur présent lors de la séquence; $A$ : acteur absent lors de la séquence; EAC : acteur ayant réalisé un entretien d’autoconfrontation suite à la séquence.

\section{Traitement des données}

Pour formaliser les règles apprises et/ou suivies par les acteurs au cours des différentes séquences du dispositif, nous avons adopté la procédure proposée par Chaliès, Bruno, Méard et alii (2010) et Chaliès, Amathieu et Bertone (2013). Les données recueillies ont été traitées en quatre étapes successives:

- l'ensemble des verbalisations enregistrées lors des séquences du dispositif et des EAC a été retranscrit verbatim. Chaque retranscription a ensuite été découpée en unités d'interaction. Ces unités ont été délimitées à partir de l'objet des significations attribuées par l'acteur autoconfronté aux événements visionnés. Une nouvelle unité d'interaction a été créée à chaque fois que l'objet de la signification attribuée par l'acteur changeait;

- pour chaque unitéd'interaction, les éléments d'étayage de la signification attribuée par l'acteur autoconfronté ont été ensuite identifiés. Par convention, ces éléments d'étayage correspondaient à l'ensemble des circonstances évoquées par l'acteur pour expliquer au chercheur la façon de s'y prendre pour signifier de la même façon que lui, c'est-à-dire en suivant la même règle, les événements de la situation de formation visionnés;

- pour chaque unité d'interaction, la règle suivie par l'acteur pour comprendre son expérience et en juger a été formalisée. Par convention, chaque règle a été étiquetée à partir de a) l'objet de la signification attribuée par l'acteur, b) l'ensemble des circonstances évoquées par l'acteur pour étayer cette signification et c) les résultats constatés et/ou attendus. Dans sa forme, chaque règle a été présentée ainsi : ["Objet de la signification» vaut dans les circonstances où «ensemble des circonstances évoquées pour étayer la signification» obtient comme résultat «ensemble des résultats constatés et/ou attendus»]. Afin de minimiser les interprétations du chercheur, chacune des règles a été étiquetée au plus près du vocabulaire des acteurs;

- la validité des résultats obtenus a été testée. L'ensemble du corpus a en effet été analysé de façon séparée par deux chercheurs. Tous les résultats obtenus ont ensuite été comparés et discutés par les deux chercheurs jusqu'à l'obtention d'un accord. En cas de désaccord, ils ont été rejetés.

\section{Résultats}

Les résultats développés ci-dessous ont été sélectionnés pour illustrer par des données empiriques les postulats constitutifs de la conceptualisation théorique préalablement présentée. Plus spécifiquement, ils permettent de mettre en évidence que les séquences de formation lors desquelles les formateurs ont exploité la vidéo (S2 et S4) ont, dans certaines circonstances, alimenté la pratique de classe des EN (S3 et S5). À plusieurs reprises lors de leur pratique de classe, ces derniers ont en effet suivi des règles qui leur avaient été enseignées et expliquées par les formateurs lors d'une séquence de formation antérieure (voir tableau 2).

Nous avons fait le choix de détailler ci-dessous l'un de ces suivis (règle 1). Il a été sélectionné car il est représentatif du suivi le plus rencontré (règles 1, 4, 7 et 11) parmi les résultats obtenus. 
Tableau 2. Identification des règles objet de l'activité de chaque acteur lors de chacune des séquences du dispositif de formation

\begin{tabular}{|c|c|c|c|c|c|c|c|c|c|c|c|c|c|c|c|}
\hline \multicolumn{16}{|c|}{ Séquences de formation } \\
\hline & \multicolumn{3}{|c|}{ Séquence 1} & \multicolumn{3}{|c|}{ Séquence 2} & \multicolumn{3}{|c|}{ Séquence 3} & \multicolumn{3}{|c|}{ Séquence 4} & \multicolumn{3}{|c|}{ Séquence 5} \\
\hline $\begin{array}{l}\text { Règles } \\
\text { (R) }\end{array}$ & FU & FT & EN & FU & FT & EN & FU & FT & EN & FU & FT & EN & FU & FT & EN \\
\hline $\mathrm{R} 1$ & - & $\mathrm{S}$ & - & $\mathrm{EO} / \mathrm{EX}$ & $\mathrm{EO} / \mathrm{EX}$ & $S$ & - & - & App & - & - & - & - & - & $\ln$ \\
\hline R2 & - & $\mathrm{S}$ & - & - & - & - & - & - & - & - & - & - & - & - & - \\
\hline R3 & - & $\mathrm{S}$ & - & - & - & - & - & - & - & EO & EX & $\mathrm{S}$ & - & - & App \\
\hline R4 & - & $\mathrm{S}$ & - & $\mathrm{EO} / \mathrm{EX}$ & $\mathrm{EO} / \mathrm{EX}$ & $S$ & - & - & App & - & - & - & - & - & - \\
\hline R5 & - & $\mathrm{S}$ & - & EO/EX & EX & $S$ & - & - & $S$ & - & - & - & - & - & - \\
\hline R6 & - & $\mathrm{S}$ & - & - & - & - & - & - & S & - & - & - & - & - & - \\
\hline R7 & - & $\mathrm{S}$ & - & $\mathrm{EO} / \mathrm{EX}$ & EO/EX & $S$ & - & - & App & - & - & - & - & - & In \\
\hline R8 & - & $\mathrm{S}$ & - & EO & EX & $\mathrm{S}$ & - & - & $\mathrm{S}$ & EX & - & $\mathrm{S}$ & - & - & $\mathrm{S}$ \\
\hline R9 & - & $S$ & - & - & - & - & - & - & - & $\mathrm{EO} / \mathrm{EX}$ & EX & $S$ & - & - & App \\
\hline $\mathrm{R} 10$ & - & $\mathrm{S}$ & - & - & - & - & - & - & - & - & - & - & - & - & - \\
\hline $\mathrm{R} 11$ & - & $S$ & - & EO/EX & EO/EX & $S$ & - & - & App & - & - & - & - & - & In \\
\hline
\end{tabular}

Légende : ce tableau présente, pour chacune des séquences constitutives du dispositif de formation, la nature de l'activité des acteurs en fonction des règles. Les acteurs peuvent mener une activité de suivi de la règle en contexte de travail (S). Cette activité peut aboutir pour les EN à un apprentissage de la règle considérée (App), voire à son interprétation (In). Les formateurs (FT et FU) peuvent aussi exploiter la règle comme objet de leur enseignement ostensif (EO) et/ou de leurs explications ostensives (EX).

À l'instant considéré de la leçon (S3), un des EN et le FT étaient positionnés sur le bord de la piste d'athlétisme, environ à mi-longueur des couloirs aménagés avec des haies basses. Ils observaient ensemble les élèves au travail. L'EN a jugé que les élèves s'exerçant dans le premier couloir ne réalisaient pas correctement ce qui leur avait été demandé. Il a formulé au formateur les raisons de cette difficulté puis les consignes qui selon lui seraient à délivrer pour aider les élèves :

\section{Extrait 1. Extrait de la séquence 3 de formation}

EN : Là en fait en 1, ils [les élèves] ne s'équilibrent pas. Ils pensent à partir loin de la haie. Ça commence à rentrer. Après sur leur position, sur s'équilibrer, ils n'y pensent pas.

FT : Sur l'équilibration après l'attaque?

EN : Oui, une fois qu'ils ont impulsé, ils pensent au pied d'impulsion comme demandé mais après c'est fini... Les bras c'est le bazar. Le tronc aussi... II faut revenir sur les bras, pour qu'ils reviennent comme ça [I'EN mime l'action des bras à réaliser]. Sinon ils ne vont pas y penser. Ils sont là [l'EN mime le positionnement des bras tendus et des rotations du tronc]...
EN : Là, je vais les arrêter juste eux... Je remontre et ils s'y remettent. On ne perd pas de temps...

Lors de son EAC, I'EN a justifié son action auprès du FT. L'extrait qui suit permet d'identifier la règle qu'il a suivie lors de la leçon pour s'engager dans l'interaction avec ce dernier :

\section{Extrait 2. Extrait de l'entretien d'autoconfrontation} de l'EN à propos de la séquence 3 de formation

$\mathrm{EN}$ : Je répète en fait là. Ils [les formateurs] nous l'ont appris ça...

Chercheur (Ch) : Répéter?

EN : Oui. Je redis que le franchissement c'est l'impulsion, bon déjà attaquer loin [de la haie] et là les élèves c'est pas mal... Et puis aussi l'équilibration. Donc comment travailler pendant le franchissement pour s'équilibrer. Les bras.

Ch : Tu dis qu'ils vous l'ont appris...

EN : On a vu ça à la vidéo la dernière fois [séquence 2]. Comment les bras doivent être placés à mi-hauteur et en opposition pour maintenir le tronc droit... S'équilibrer pour rester dans l'axe et pas perdre de vitesse. 
Lors de cet extrait d'EAC, I'EN a formulé la règle qu'il avait suivie en classe pour interpréter l'activité des élèves. Ainsi, selon lui : ["S'équilibrer lors du franchissement de la haie» vaut pour «placer les bras à mihauteur et en opposition» et «maintenir la tête fixe» ce qui obtient comme résultats de "rester dans l'axe» et «ne pas perdre de vitesse»]. Cet extrait permet en outre d'accéder à l'histoire des usages de cette règle par cet EN et les formateurs. Cette règle, suivie par I'EN en classe, a été initialement ("la dernière fois ») enseignée par les formateurs par l'intermédiaire d'extraits vidéo lors de la séquence de formation menée à l'université (S2). L'extrait suivant permet de confirmer que l'apprentissage de la règle considérée a bien eu lieu lors de cette séquence. II permet par ailleurs de situer que cet apprentissage fait suite à une activité d'enseignement ostensif menée par les formateurs. En mettant en exergue l'interpénétration de cette dernière avec une activité d'explication, cet extrait invite toutefois à associer avec prudence l'apprentissage de la règle à la seule activité d'enseignement des formateurs :

\section{Extrait 3. Extrait de la séquence 2 de formation}

FT : Là on voit le franchissement... On en a parlé en classe [séquence 1] mais ce n'était pas flagrant... Beaucoup n'avaient rien vu...

FU : Et oui donc on va s'arrêter sur ça. S'équilibrer lors du franchissement après l'impulsion, OK?

EN : Mm.

FU : Donc là on a pris le passage de Magalie [une élève] et on voit comment s'équilibrer... [L'extrait vidéo est lancé].

FU : Voilà j'arrête là [il réalise un arrêt sur image]. Là elle franchit. Là c'est bon, on peut dire qu'elle s'équilibre. Alors pourquoi?

EN : Mm.

FT : Moi je regarde un truc très précis. Un, les bras. Là, ils sont à mi-hauteur. OK c'est bon. On est pas dans les bras en bas du corps comme on a vu [il démontre avec les bras le long du corps]. Là ils sont en opposition. Bien comme ça [il démontre avec un bras devant et l'autre derrière]. Là c'est fixe. D'accord?

EN : Oui.

FU : Si ça c'est fait alors ils vont courir dans l'axe et, et donc ils perdent moins de temps. Donc là si je lance [l'extrait vidéo est relancé], on regarde sur la deuxième haie... Là, j'arrête [il réalise un arrêt sur image]. C'est pareil le franchissement est bon.

FT : Et elle ne décélère pas... Elle s'équilibre par les bras, là à mi-hauteur, un devant, un derrière...

Comme spécifié en amont, cet extrait permet de situer la complexité de l'activité de formation des formateurs pour faire apprendre la règle aux EN. À cet instant, le FU a en effet exploité la vidéo pour s'engager dans un enseignement plus ostensif que n'avait pu le faire le FT lors de la formation menée en classe (S1) ( $O \mathrm{On}$ en a parlé mais ce n'était pas flagrant en fait»). Le FU, aidé par le $\mathrm{FT}$, a plus précisément réalisé le dressage ostensif ${ }^{4}$ du lien de signification entre d'une part, l'énoncé de la règle ("S'équilibrer lors du franchissement de la haie») et d'autre part des circonstances expérientielles jugées comme exemplaires et données à observer à la vidéo. Pour ce faire, il a procédé à plusieurs arrêts sur image ou ralentis afin de rendre le plus explicite possible «un truc très précis » qu'il donne à observer et qui est constitutif de ce qu'il entend par «s'équilibrer lors du franchissement de la haie». Autrement dit, la règle suivie par l'EN pour interpréter l'activité des élèves en classe et agir en conséquence lui a bien été enseignée lors de la séquence de formation précédente.

Il convient de noter que la règle considérée n'avait pas été seulement enseignée aux EN. Elle leur avait été aussi plus ou moins simultanément expliquée. Les formateurs ont en effet cherché à immédiatement accompagner les premiers suivis de la règle enseignée par les EN en s'efforçant d'écarter les différents malentendus possibles. Pour ce faire, ils ont multiplié les exemples constitutifs de la règle étiquetée afin de parvenir à fonder un accord avec les EN quant à ce que pourrait être «S'équilibrer lors du franchissement de la haie» de façon exemplaire. Outre l'appui sur l'observation par les EN des images fournies par les extraits vidéo, les formateurs ont aussi procédé à plusieurs formulations des critères donnés à observer (« je regarde un truc très précis. Un, les bras. Là, ils sont à mi-hauteur [...] puis en opposition [...] c'est fixe »). Ils se sont aussi attachés à les démontrer. Ils ne se sont donc pas satisfaits du seul enseignement de la règle. Ils se sont engagés dans une explication de cette dernière qui a, sans doute, aussi contribué à son apprentissage par certains EN. Le retour sur l'EAC du FT permet de préciser ce résultat :

4 Wittgenstein n'associe pas le mot «dressage » à une quelconque méthode d'éducation cynique mais plutôt à une «intellection philosophique fondamentale» de la constitution des liens de signification établis entre diverses expériences. Lors de l'enseignement ostensif de toute nouvelle règle, les formateurs s'engagent en effet théoriquement dans une activité de monstration assimilable à un "dressage ostensif» d'un lien de signification (voir par exemple Laugier, 2009) entre, d'une part, des circonstances expérientielles identifiées, montrées, décrites, voire données à vivre, et, d'autre part, l'expérience langagière visant à les nommer, c'est-à-dire l'énoncé de la règle et enfin, les résultats attendus dans le cas d'une pratique en conformité avec celle-ci. 
Extrait 4. Extrait de l'entretien d'autoconfrontation du FT à propos de la séquence 2 de formation

FT : Là on reprend ce que c'est que s'équilibrer. J'en ai déjà parlé la fois d'avant [séquence 1] mais on y revient ensemble [avec le FU] parce qu'on est un peu passé à côté.

Ch: À côté?

FT: Ils [les EN] ne l'ont pas vu. J'ai essayé de le montrer comme là à la vidéo mais en direct [en classe] ce n'est pas... Je n'avais pas ce que je voulais au moment où je le voulais. Je le voyais mais eux [les EN] étaient sur autre chose.

Ch : Donc... là c'est?

FT : Là c'est s'équilibrer lors du franchissement. Ch : Et tu...

FT: Là l'idée c'est qu'ils situent clairement ce que c'est que bien s'équilibrer...

Ch : Et donc...

FT : Là c'est plus simple... II [le FU] les centre sur l'élève dont on sait... on est sûr que c'est bon et hop au moment du franchissement il arrête [la vidéo].

Ch: Plus simple?

FT : Oui on contrôle. On avait vu que Magalie [l'élève observée] allait correctement le faire. Y a pas de surprise. En plus on peut arrêter, on remet, on va un peu plus loin... On ne peut pas le rater là avec la vidéo alors qu'en classe avant que tu tombes sur le bon exemple ce n'est pas gagné quoi... En plus on peut insister.

Ch : Donc c'est mieux là?

FT : Il [le FU] laisse quand même la vidéo passer. Au début, il arrête. Après on essaye de vite passer à du ralenti et puis à vitesse normale... En classe c'est pas pareil. Ils ne vont pas pouvoir arrêter les élèves, leur demander de repasser en refaisant la même chose... La vidéo c'est bien mais c'est quand même loin... II faut prendre des précautions...

Lors de cet extrait d'EAC, le FT a révélé au chercheur le caractère paradoxal de l'utilisation de la vidéo lors de l'enseignement de règles aux EN. D'un côté, cette utilisation lui a permis de sortir des difficultés qu'il avait pu rencontrer lors de la situation de formation en classe ("Je n'avais pas ce que je voulais au moment où je le voulais»). L'usage de la vidéo a donc simplifié l'activité d'enseignement des règles («c'est plus simple») notamment parce qu'ils ont pu « contrôler» avec le FU ce qu'ils voulaient donner à observer aux EN, c'est-à-dire la nature des exemples associés à l'étiquette de la règle. Contrairement à ce qui a pu se passer lors de la séquence 1 ("en classe avant que tu tombes sur le bon exemple c'est pas gagné»), les formateurs ont pu effectivement sélectionner les exemples d'activité d'élèves considérés comme pertinents pour la formation avant même de s'engager dans leur activité d'enseignement ( $\mathrm{Y}$ a pas de surprise tère ostensif de leur enseignement en tirant profil du caractère "sans surprise» des exemples d'activité d'élèves enregistrés. Lors de son EAC, le FT a ainsi précisé que les EN ne pouvaient «plus rater » ce que lui et le FU souhaitaient leur montrer parce qu'ils pouvaient insister en procédant à des arrêts sur image, à des ralentis ou en repassant plusieurs fois le même extrait. Complémentairement, les arrêts sur image leur ont aussi permis de s'engager collectivement dans une explication de la règle préalablement enseignée. Ils ont alors suivi la règle [« Insister auprès des $\mathrm{EN}$ » vaut pour «ils [les élèves] le voient, on leur dit» et parfois "on démontre», ce qui obtient comme résultat que les EN "choppent» la règle enseignée].

D'un autre côté, le FU a, quant à lui, précisé que I'utilisation de la vidéo nécessite de prendre des précautions pour parvenir à «préparer quand même le travail en classe » :

\section{Extrait 5. Extrait de l'entretien d'autoconfrontation} du FU à propos de la séquence 2 de formation

Ch : Peut-on s'arrêter sur ce moment-là?

FU : Oui, là c'est quand même toutes les précautions qu'il faut prendre avec l'outil vidéo.

Ch : Là tu prends des précautions, c'est ça? Tu peux m'en dire un peu plus?

FU: Ouij'y suis contraint en fait. Sinon, et c'est presque paradoxal... le but c'est quand même de préparer le travail en classe. Donc il faut quand même parvenir à rapprocher les extraits vidéo de ce qui se passe réellement en classe.

Ch : Et donc tu dis «je prends des précautions pour cela». C'est bien ça?

FU : Oui, c'est rien de très... Mais bon là par exemple je passe vite à du ralenti pour qu'ils [les EN] voient quelque chose et puis à la vitesse normale...

Ch : qui est la vitesse...

$\mathrm{FU}$ : de ce qui se passe réellement en classe oui.

Lors de cet extrait d'EAC, le FU a plus précisément relevé que l'observation de l'activité des élèves ne pouvait se faire de façon aménagée en classe comme il s'y était autorisé en formation avec la vidéo. En ce sens, l'exemple d'activité constitutif de la règle donné à observer par l'intermédiaire de la vidéo pouvait parfois si aucune précaution n'était prise rester inutilisable par les EN car trop éloigné du contexte de classe. Selon le $\mathrm{FU}$, il est donc nécessaire dès la formation de se rapprocher en quelque sorte des circonstances de classe pour permettre ensuite les suivis par les EN des règles enseignées. II a alors suivi la règle [«Prendre des précautions dans l'utilisation de la vidéo " vaut pour "passer vite à du ralenti et puis à vitesse normale», ce qui obtient comme résultat de «rapprocher les extraits 
vidéo de ce qui se passe en classe»]. L'extrait d'autoconfrontation d'un des EN relatif à la même séquence de formation permet aussi de mettre en lumière ce caractère paradoxal de l'utilisation de la vidéo :

Extrait 6. Extrait de l'entretien d'autoconfrontation de l'EN à propos de la séquence 2 de formation

EN : On voit bien là ce que c'est que s'équilibrer. On peut le voir, le revoir... Donc à la fin c'est clair.

$\mathrm{Ch}: \mathrm{C}^{\prime}$ est intéressant donc?

EN : Oui, on apprend ce que c'est avant de... de l'utiliser... Après c'est plus compliqué...

$\mathrm{Ch}$ : Compliqué?

EN : Oui en classe quand on essaye... Là c'est cool... si tu rates ça repasse... Tu peux aussi le voir comme là au ralenti... En classe, c'est pas pareil... T'as pas le temps comme là de te préparer. Ça va vite, c'est jamais pareil... C'est plus dur de voir. En plus t'es jamais bien placé, de profil. Là, tu peux voir si les bras c'est bon à mi-hauteur..

L'analyse de cet extrait d'EAC permet de mettre en exergue trois principaux résultats. Tout d'abord, I'EN a jugé la modalité de formation mise en œuvre par les formateurs « intéressante » et «claire». Il a alors suivi la règle [«Apprendre ce que c'est que s'équilibrer» vaut pour «dire vous allez voir ce que s'est que s'équilibrer» et le donner à «voir » et « revoir »]. D'autre part, il a souligné que l'utilisation en classe de ce qui a été appris en formation grâce notamment à la vidéo est «plus compliquée». En formation, I'observation des élèves s'est révélée différente de celle effectuée en classe. L'EN a plus précisément formulé la règle qu'il a suivie pour porter ce jugement. Ainsi, selon lui, I'observation en formation était plus "cool» car il était dans de bonnes conditions pour observer («bien placé en fait, de profil»). D'autre part, il pouvait «se préparer» à observer ce qui lui avait été préalablement annoncé. Enfin, en situation de formation exploitant la vidéo, il pouvait «voir au ralenti » l'activité des élèves et s'il n'y parvenait pas, il pouvait la visionner à nouveau («si tu rates ça repasse»).

Même s'il relève comme les formateurs que les circonstances de classe sont par nature toujours singulières, I'EN justifie toutefois une nouvelle fois sa pratique de classe auprès des élèves en fin de dispositif (S5) à partir de ce qu'il a pu vivre lors d'une séquence de formation antérieure :

Extrait 7. Extrait de l'entretien d'autoconfrontation de l'EN à propos de la séquence 5

Ch : Là, je me trompe peut-être mais tu n'es plus exactement sur ce qu'ils [les formateurs] vous avaient dit initialement, non?
EN : À propos de l'équilibration?

Ch: Oui quand ils [les élèves] franchissent? Là il me semble que tu te limites plus à ce qu'ils vous avaient présenté comme critères?

EN : Bien si quand même les bras. Être en opposition.

$\mathrm{Ch}$ : C'est sur cela que tu reviens là?

EN : Oui. Enfin sur une opposition aménagée.

Ch : Aménagée c'est..

EN : Dans le sens où là Élise [une élève en difficulté] je peux pas lui demander d'être complètement en opposition.

Ch : Mais en même temps c'est ce qui te guide non? Dans ce que tu regardes? Par rapport à ce qu'ils [les formateurs] avaient dit.

EN: Oui mais on peut quand même en sortir si tu veux. C'est ce qu'on a vu quand même. T'es obligé d'adapter un peu. À vitesse plus réduite l'opposition est moins importante en fait... Y a moins de déséquilibres.

$\mathrm{Ch}$ : Tu dis que vous l'avez vu ça?

EN : Oui. La dernière fois avec plein d'extraits qui nous ont posé des problèmes... justement pour aménager en fait un peu... en fonction des élèves.

Cet extrait d'EAC est d'autant plus significatif qu'il permet de mettre en évidence une activité d'interprétation par l'EN de la règle préalablement apprise. Lorsqu'il signifie son activité de classe, il suit en effet cette dernière tout en procédant à un usage extensif («on peut quand même en sortir») du lien de signification («T'es obligé d'adapter un peu. À vitesse plus réduite l'opposition est moins importante... Y a moins de déséquilibres») que les formateurs lui avaient initialement enseigné et qu'il avait appris. Ainsi, selon lui, ["S'équilibrer lors du franchissement de la haie» vaut pour «placer les bras à mi-hauteur et en opposition plus ou moins complète selon le niveau de pratique» et «maintenir la tête fixe», ce qui obtient comme résultats de «rester dans l'axe» et «ne pas perdre de vitesse»]. Cet extrait permet en outre d'accéder pour ainsi dire à l'origine de cette activité d'interprétation de la règle par cet EN. Dans la situation de classe considérée, la règle est suivie une nouvelle fois par l'EN. Ce suivi interprété de la règle fait suite («la dernière fois») à un travail d'interprétation mené avec les formateurs lors de la séquence de formation menée à l'université (S4), travail lors duquel ces derniers avaient exploité "plein d'extraits vidéo qui posaient des problèmes ».

\section{Discussion}

L'idée n'est pas ici de proposer une discussion construite à partir d'une généralisation des résultats obtenus dans le cadre d'une étude de cas et ce même si leur récurrence dans plusieurs autres études pourrait 
permettre de l'envisager (par exemple : Gaudin, Flandin, Ria et al., 2014, Gaudin \& Chaliès, 2016). Les résultats obtenus dans le cadre de l'étude de cas mériteraient en effet d'être validés par des travaux menés avec des échantillons scientifiquement plus significatifs. De la même manière, le contexte de l'enseignement de l'EPS revêt des singularités fortes (par exemple la possibilité offerte aux formateurs et aux EN de respectivement donner à observer ou d'observer les activités menées par les élèves et leurs évolutions lors du temps de classe) et il serait en ce sens nécessaire de réaliser un tel dispositif de formation auprès d'EN d'autres disciplines scolaires.

Dans la continuité de l'objet premier de l'article, cette discussion cherche plutôt à s'appuyer sur ces résultats pour illustrer un certain nombre de postulats théoriques et mettre en exergue le caractère heuristique de la conceptualisation théorique retenue pour réinterroger la principale controverse subsistant dans la littérature du domaine relative aux modalités «normative» ou "développementale» d'utilisation de la vidéo au sein des dispositifs de formation proposés aux EN. Plus spécifiquement, elle cherche à alimenter une réflexion théorique plus large sur comment l'utilisation de la vidéo pourrait être pensée pour contribuer à la construction des sujets professionnels en formation.

\section{Outil vidéo et apprentissage des règles : une nécessaire entrée "normative » à visée de développement}

Lors du dispositif de formation étudié, les EN ont finalement appris six des huit règles qui leur ont été enseignées et/ou expliquées par les formateurs. À I'issue du dispositif de formation, les EN ont été en effet, d'une part, en capacité d'«être gouvernés » par ces règles dans leur pratique de classe. Ils sont de ce fait parvenus à obtenir les résultats attendus que leur avaient enseignés initialement les formateurs. Les EN ont été, d'autre part, capables de s'engager dans des suivis adéquats de ces règles pour indiquer les raisons (Chauviré, 2004) de leurs actions aux formateurs (lors des séquences de formation) ou au chercheur (lors des EAC) qui les interrogeaient.

Ce résultat permet d'ouvrir la discussion sur les circonstances de formation ayant permis cet apprentissage de nouvelles règles par les EN. Plus précisément, il permet de mettre en avant le caractère productif d'une modalité «normative» singulière d'utilisation de la vidéo
Lors de la S2 menée à l'université, les formateurs ont exploité la vidéo pour renforcer le caractère ostensif de leur activité d'enseignement de toute nouvelle règle. Ils ont plus précisément cherché à renforcer le caractère ostensif de la définition du lien de signification qu'ils établissaient entre, d'une part, l'énoncé des règles enseignées et, d'autre part, des circonstances expérientielles sélectionnées et enregistrées lors de la leçon et données à visualiser. Assez paradoxalement, ils souhaitaient ainsi sortir, en s'appuyant sur les possibilités offertes par la vidéo, des difficultés rencontrées lors de la situation de formation menée en classe (S1) au cours de laquelle le FT n'avait pas pu réellement donner à observer aux EN les exemples souhaités au moment opportun. Autrement dit, en procédant à son aménagement par l'enregistrement et la sélection d'extraits vidéo singuliers, les formateurs ont cherché à s'extraire de la pratique de classe pour au final mieux y préparer les EN.

Une analyse des résultats obtenus permet de situer que les formateurs se sont plus précisément engagés dans des usages singuliers des extraits vidéo enregistrés lors de la leçon pour en quelque sorte aménager la pratique de classe et ainsi s'assurer de l'apprentissage de certaines règles par les EN. Ils ont cherché, tout d'abord, par leur exploitation de la vidéo, à «contrôler » la nature des exemples donnés à observer à ces derniers suite à l'énonciation des règles (Masats \& Dooly, 2011). Contrairement à ce qui se joue généralement lors d'une formation par l'observation de pratiques professionnelles en classe (Lasagabaster \& Sierra, 2011), les formateurs ont pu effectivement sélectionner par avance les exemples extraits de leçons qu'ils jugeaient comme les plus pertinents en termes d'aspects constitutifs des règles enseignées. Ils ont ainsi sélectionné les franchissements des élèves jugés comme les plus adéquats au cœur de la leçon pour étayer leur enseignement des règles. Ils ont par ailleurs exploité la vidéo pour renforcer le caractère exemplaire de l'activité des élèves donnés à observer (Santagata, 2014). Pour ce faire, ils ont insisté sur les exemples visualisés en les présentant aux EN de façon aménagée. Ils ont alors procédé à de multiples reprises à des arrêts sur images, ils ont usé du ralenti et proposé plusieurs visionnages successifs (Tripp \& Rich, 2012).

Les résultats de cette étude mettent aussi en exergue toute la complexité pour les EN de se saisir de certains types d'exemples exemplaires (par exemple l'observation de la pratique effective de classe du FT en séquence 1) et, par-là même, donnent à voir les inté- 
rêts de l'usage de la vidéo en formation. Les formateurs se sont en effet appuyés sur les possibilités offertes par la vidéo pour rendre accessibles aux EN les exemples exemplaires des règles lors de l'enseignement ostensif. En ce sens, ils ont procédé à des arrêts sur images, à des visionnages répétés ou à des ralentis pour accompagner de leurs propres formulations, voire parfois de leurs propres démonstrations de comportements d'élèves, certains critères donnés à observer. Les formateurs se sont par ailleurs servis de la vidéo pour s'engager dans des explications ostensives afin de lever les malentendus, les mésinterprétations et/ou les incompréhensions des EN révélés lors de leurs premiers suivis des règles qui leur avaient été préalablement enseignées. Pour ce faire, ils ont associé à l'exemple exemplaire initialement enseigné d'autres exemples qui, de par leur multiplicité et leurs traits communs (Narboux, 2003), ont permis aux EN de se saisir de l'exemplaire (Laugier, 2009). À ce titre, l'outil vidéo est donc aussi apparu comme particulièrement intéressant pour les formateurs qui ont pu sélectionner par avance la nature de ces exemples mais aussi programmer leur agencement au moment du visionnage (par exemple en les présentant du plus « ressemblant» au moins «ressemblant» à l'exemple initialement enseigné) afin que les EN puissent s'en saisir.

À ce stade de développement, deux points théoriques peuvent être abordés. Déjà abordé dans d'autres champs théoriques comme la philosophie analytique, le premier point de discussion fera sans doute l'objet d'autres travaux en sciences de l'éducation et de la formation. Il se situe en effet autour du paradoxe selon lequel, d'un côté, il semble possible de considérer l'existence d'un "primat des exemples exemplaires» (Narboux, 2003) constitutifs des règles enseignées tandis que, de l'autre, il n'est pas possible de faire l'économie de constater que leur choix tient toujours de l'arbitraire (Chauviré, 2003; Laugier \& Chauviré, 2006). Les exemples associés aux règles enseignées ne sont en effet que des préférences auxquelles les formateurs octroient le statut d'exemples «exemplaires de... ». Pour autant, ces exemples se doivent d'être par nature "paradigmatiques ou canoniques» (Laugier \& Chauviré, 2006; Narboux, 2003) pour les règles professionnelles considérées sans quoi, ne fixant aucune référence pédagogique (Le Blanc, 1998) source ensuite de développement pour les EN, ils ne seraient pas d'une grande utilité. C'est en ce sens qu'il est possible de considérer qu'ils jouent « un rôle vital » (Davis, 2009), notamment lorsqu'il s'agit de questionner les usages des règles inhérents à leur enseignement initial (Cometti, 2004). Les exemples exemplaires deviennent alors en effet les « aspects 》 (Chauviré, 2010) des règles professionnelles enseignées dont les formés doivent nécessairement se saisir pour apprendre ces dernières, c'est-à-dire être en capacité de les suivre a posteriori dans de nouvelles circonstances de formation et/ou de travail.

Le second point théorique relève davantage d'une mise en discussion de la modalité «normative » d'utilisation de la vidéo déployée à partir des postulats de l'anthropologie culturaliste avec d'autres modalités elles-mêmes fondées à partir d'autres lectures théoriques s'inscrivant pour autant dans une seule et même approche de la vidéoformation "centrée activité». À l'évidence, nous sommes en adéquation avec les propos tenus par Flandin, Leblanc et Muller (2015, p. 193) lorsqu'ils avancent que le développement de l'activité enseignante est « un processus dynamique produisant dans l'action des compromis opératoires qui combinent d'une part ce qu'on demande de faire au sujet à partir de prescriptions descendantes et remontantes et d'autre part ce que ça lui demande pour réaliser de tels compromis [...]». Tel que nous avons pu l'expliciter et l'illustrer, le développement de l'activité s'inscrit effectivement dans un double mouvement d'apprentissage puis de développement sans cesse enrichi par les circonstances situationnelles rencontrées. À ce titre, nous sommes d'accord avec ces auteurs lorsqu'ils stipulent que «l'activité [ne peut être] pensée comme un produit achevé qui est à atteindre» (p. 193).

Par contre, nous nous situons en désaccord lorsqu'il s'agit d'identifier et de formaliser les actions de formation qui ont potentiellement initié ce développement. Alors que Flandin, Leblanc et Muller (2015) considèrent que «la pertinence [des questions ayant émergé en formation] est dictée par les significations construites par les acteurs eux-mêmes» (p. 193), il ne semble pas possible, du point de vue de l'anthropologie culturaliste, d'envisager comment ces «acteurs» (ici les EN) parviennent à signifier «correctement» (au sens où il est attendu de le faire dans la communauté professionnelle considérée) les circonstances vécues de formation ou de travail sans avoir été en amont instruits par les formateurs. Autrement dit, les propositions de ces auteurs nous paraissent sous-estimer pour ainsi dire le poids des activités engagées par les formateurs (activités d'enseignement, d'étayage, d'explications, etc.) compte tenu de leur projet intentionnel de formation et, par voie de conséquence, surestimer l'hypothèse d'un 
primat accordé aux EN dans l'activité de construction de nouvelles significations professionnelles. Les propos tenus par Durand sur lesquels s'appuient ces auteurs sont d'ailleurs à ce titre très explicites : «l'asymétrie du couplage structurel fait que c'est l'acteur qui définit ce qui est significatif/pertinent pour lui : ce n'est donc pas l'environnement du formé, dont fait partie le formateur et le dispositif de formation, qui spécifie son activité» (2008, p. 12). L'idée n'est pas ici de nier chez les acteurs (y compris ceux en formation) l'existence de dispositions à signifier les faits et occurrences de leur contexte de travail de façon immédiate et préréflexive (Legrand, 2007). Bon nombre de significations culturelles sont en effet transmises et appropriées dans des situations et des activités de transmission informelles par l'intermédiaire d'interactions non verbales et/ou d'alignements informels avec la pratique d'autres acteurs (Lave \& Wenger, 1991 ; Rogoff, Matusov \& White, 1996). Toutefois, comme le précise Taylor, ce type de transmission aboutit à une "compréhension non formulée» du monde dans lequel agissent les acteurs et leur «permet [seulement] de trouver un sens aux choses et aux actions [...] entièrement informulé» $(1995$, p. 560). Autrement dit, il y a bien la possibilité d'une appropriation mais à partir d'un régime de réflexivité qui ne permet pas ensuite aux acteurs de se représenter et de rendre compte des raisons de leurs actions et donc des règles qu'ils « suivent » lorsqu'ils agissent (Ogien, 2007). N'ayant effectivement pas appris, par l'intermédiaire de situations singulières de formation au cœur desquelles les formateurs s'engagent dans un enseignement ostensif des règles et les accompagnent par des réélaborations discursives et réflexives, à mettre en correspondance les énoncés des règles de l'action et la réalisation des actions réglées, ils en restent à la seule acquisition de capacités pratiques à réaliser des actions "gouvernées» par des règles (Ogien, 2007).

Par-delà le contexte même de la vidéoformation, c'est ici un point de discussion significatif entre deux lectures fondamentales du développement humain. D'un côté, la théorie sémiologique du cours d'action s'inscrit effectivement dans le cadre d'une épistémologie énactive issue de l'hypothèse de «l'autopoïèse» (Varela, 1989) selon laquelle les systèmes vivants, quel que soit leur degré d'organisation et de sophistication, s'autoproduisent, c'est-à-dire produisent et modifient eux-mêmes leur propre structure en fonction des perturbations qui proviennent de leur environnement. Chaque acteur possède ainsi, selon cette lecture, des dispositions anthropologiques, en particulier celle de typicalisation de ses propres expériences vécues au gré des circonstances, lui permettant de construire de nouvelles significations dans un couplage répété avec sa situation et de se développer à partir d'elles. D'un autre côté, l'anthropologie culturaliste, comme d'autres lectures culturalistes avant elle (c'est le cas par exemple de la psychologie historico-culturelle développée par L. Vygostki), adopte une conception «allopoïétique» (Descombes, 2004) pour étudier la construction de la subjectivité. Selon cette conception, les significations culturelles sont "constitutives» (Chauviré, 2009) de la subjectivité. Elles nécessitent donc d'être initialement enseignées pour, une fois apprises, pouvoir être source de développement de chaque acteur qui se les auto-adresse. Autrement dit, dans le cadre de la formation des EN, chacune des règles constitutives du métier peut donc être initialement considérée comme la source de l'activité individuelle des EN au sein des situations d'apprentissage. Elle devient ensuite potentiellement une ressource pour le développement des EN lorsque, ayant été apprise, elle est interprétée pour agir singulièrement dans la situation de pratique professionnelle rencontrée. Pour conclure, Flandin, Leblanc et Muller (2015), en s'appuyant sur la théorie sémiologique du cours d'action, soutiennent donc qu'une modalité « développementale» d'utilisation de la vidéo peut être à l'origine du développement de l'activité des EN. Les résultats obtenus dans cette étude, prenant appui sur le cadre de l'anthropologie culturaliste, permettent de soutenir qu'une modalité «normative» doit y être nécessairement associée. Plus exactement, ils permettent de poser I'hypothèse d'un primat de la modalité «normative » sur la modalité « développementale» dans l'utilisation de la vidéo pour former les EN.

\section{Outil vidéo et développement à partir des règles apprises : une nécessaire entrée « développementale » à visée de normativité}

Considérer comme nous venons de l'expliciter qu'il existe un primat de la modalité "normative» sur la modalité «développementale» dans l'utilisation de la vidéo pour former les EN ne peut en aucun cas aboutir au raccourci selon lequel la formation se réduirait alors à l'appropriation «de savoirs, de règles prédéfinies et prescrites par un formateur » (Flandin, Leblanc \& Muller, 2015, p. 193), autrement dit à l'acquisition et à l'application de «bonnes pratiques» présumées (Leblanc 
\& Veyrunes, 2011). Accepter ce primat revient plutôt à considérer que toute activité professionnelle procède selon des règles, certes initialement apprises, mais aussi et surtout interprétées (Cometti, 2004), c'est-àdire toujours adaptées à la singularité des situations rencontrées. Autrement dit, chaque EN se développe alors singulièrement par des suivis de règles toujours réitérés dans des formes originales (Bohman, 2011).

Les résultats détaillés en amont permettent justement de montrer combien il est donc essentiel d'interroger les possibles rapprochements entre, d'une part, les circonstances de formation donnant une signification initiale aux règles enseignées et expliquées, voire aux premiers suivis de celles-ci par les EN (à l'université) et, d'autre part, celles de travail en classe (Williams, 2002). Toute la difficulté pour les formateurs s'est en effet située à ce niveau. Il leur a fallu parvenir à enseigner de façon ostensive les règles et à accompagner leurs premiers suivis voire leurs interprétations par les EN au cœur de situations de formation mais aussi de travail. À ce titre, I'usage de la vidéo est apparu, notamment aux formateurs, paradoxalement tout à la fois intéressant et limitatif. Intéressant, d'une part, car ils ont pu s'en saisir pour passer progressivement d'une modalité normative à une modalité «développementale » et ainsi insister sur ce qu'ils souhaitaient donner à faire signifier aux EN selon leur intention de formation. Ainsi, lors de l'enseignement de toute nouvelle règle, les formateurs ont exploité l'outil vidéo pour rendre accessible aux EN deux des aspects la constituant, l'exemple exemplaire et les résultats attendus. Lors de l'accompagnement des premiers suivis des règles par les $\mathrm{EN}$, les formateurs ont ensuite plutôt utilisé l'outil vidéo selon une modalité « développementale » en cherchant à les confronter à d'autres exemples pouvant être associés à la règle considérée. C'est d'ailleurs dans le cadre de cette modalité qu'ils ont ensuite poursuivi leur accompagnement à visée de développement professionnel. Lors du travail mené en S4, ayant pour objectif premier d'engager les EN dans I'interprétation des règles préalablement apprises, ils ont en effet exploité l'outil vidéo pour confronter les EN à des exemples "problématiques », c'est-à-dire présentant moins de traits de similarité avec l'exemple initialement utilisé pour enseigner la règle.

Parallèlement, l'usage de la vidéo est aussi apparu aux formateurs comme limitatif. Même s'ils ne l'interprètent pas explicitement comme tel, c'est bien la difficulté rencontrée pour faire en sorte que la modalité «développementale» d'utilisation de la vidéo ouvre sur un processus de normativité chez chacun des EN qui est mise en exergue par les résultats proposés. Qu'ils soient exemplaires, multiples et proximaux ou porteurs de singularités invitant à l'interprétation, tous les extraits vidéo exploités en contexte de formation ont eu ceci en commun qu'ils ont pu ensuite rester inutilisables dans de nouvelles circonstances par nature très différentes que sont celles de la classe. Placés dans des situations de travail comportant des caractéristiques contextuelles singulières (par exemple la vitesse des événements), les EN se sont en effet dans bon nombre de cas retrouvés en difficulté pour parvenir à signifier des traits communs avec des situations de formation déjà rencontrées et in fine à s'y adapter.

Dans les résultats détaillés en amont, on peut toutefois relever que les formateurs se sont employés à prendre des précautions lors de l'accompagnement des premiers suivis des règles enseignées (S2) afin de rapprocher la situation de formation de celle de travail en classe. Ils se sont ainsi attachés, après avoir effectué des arrêts sur images ou des ralentis, à utiliser les extraits vidéo à vitesse réelle, c'est-à-dire à la vitesse à laquelle les EN devront signifier les événements observés en classe. Pareillement, ils ont aussi pris des précautions pour accompagner par la multiplication d'extraits vidéo présentant cette fois-ci des écarts à la règle initialement enseignée (S4) l'activité d'interprétation des règles préalablement apprises par les EN. Autrement dit, les formateurs ont pris un certain nombre de précautions pour permettre aux formés de «renormaliser » les règles préalablement apprises, c'est-à-dire de les «transformer sous le coup de la singularité des situations» (Lussi Borer \& Muller, 2014a, p. 131). Ils les ont en ce sens sans cesse aidés à s'écarter des règles initialement enseignées voire expliquées, sans pour autant leur permettre de se positionner hors d'elles, et de ce fait les ont autorisés à exister comme sujets (Le Blanc, 2004).

De façon complémentaire, on peut toutefois souligner que les précautions prises par les formateurs afin d'accompagner au mieux les EN dans leurs premiers suivis des règles enseignées ne relèvent jamais, réciproquement pourrait-on dire, d'un rapprochement de la situation de travail à la situation de formation. Ce point de discussion est d'autant plus significatif qu'il invite à une reconsidération nécessaire du principe de l'alternance et de ses mises en œuvre actuelles. Il ouvre, d'une certaine manière, un champ de recherche nouveau relatif à l'aménagement des situations de travail de sorte qu'elles puissent devenir de véritables situations 
de formation au travail. Comme le soulignent Durand et Filliettaz (2009), le défi est de taille. Ce n'est effectivement pas là seulement le questionnement des modalités d'optimisation de «l'approche séquentielle traditionnelle faisant succéder à une phase de formation une phase d'opérationnalisation pratique» (p.10), ou inversement, à un temps de travail une séquence de prise de recul par rapport à celui-ci (par exemple lors d'un entretien post-leçon). Les prises de position récentes, notamment d'un point de vue institutionnel, invitant par exemple à jouer sur la distanciation ou le rapprochement entre travail et formation sont à ce titre certainement encore trop timides. Sans aller jusqu'à considérer que la formation professionnelle ne puisse se faire que dans et par le travail, il apparaît finalement incontournable de rediscuter le privilège accordé au principe de l'alternance en formation professionnelle des enseignants et d'y préférer, sans doute à terme, celui de la continuité entre travail et formation.

\section{Conclusion}

Cet article avait comme objectif de réinterroger la principale controverse subsistant dans la littérature du domaine relative aux modalités d'utilisation de la vidéo au sein des dispositifs de formation proposés aux EN. Comme spécifié en amont, il conviendrait lors de prochains travaux de sortir du contexte de l'enseignement de l'EPS porteur de spécificités fortes et significatives compte tenu de l'objet étudié. Dans d'autres disciplines, la possibilité de travailler en formation à partir de l'enregistrement vidéo de l'activité effective des élèves en classe est en effet bien différente, cette dernière étant de fait plus difficilement observable.

Toutefois, sur la base d'une conception théorique singulière, cet article permet de mettre au jour le caractère limitatif de la dichotomie usuellement établie entre des modalités d'utilisation dites «normative» et

\section{Bibliographie}

BERDUCCI D. (2004). «Developmental Continuum Vygotsky through Wittgenstein: A New Perspective on Vygotsky's ». Theory Psychology, n¹4(3), p. 329-353.

BERTONE S. \& CHALIÈS S. (2015). «Construire un programme de recherche technologique sur la formation des enseignants : choix épistémologiques et théoriques». @ctivités, $\mathrm{n}^{\circ} 12(2)$, p.53-72. En ligne : <https://activites.revues. org/1088> (consulté le 17 avril 2016).
« développementale» et souligne son caractère improductif pour penser les dispositifs de formation. De façon complémentaire, il défend une articulation potentielle de ces modalités à l'échelle de chacun des dispositifs de formation. À ce titre, il alimente les premières hybridations théoriques initiées entre les lectures s'inscrivant dans une approche de la vidéoformation « centrée activité » (à ce titre l'article de Lussi Borer \& Muller [2014a] est exemplaire). Plus en détail, et c'est sans doute là son principal apport, il pose une définition singulière de la construction du sujet professionnel en formation au cœur de laquelle une place centrale est accordée à la «normativité» comme interface entre normatif et développemental. En effet, tout comme il n'est pas possible, à l'instar d'une approche strictement développementale, de considérer la construction du sujet professionnel hors de toute règle, il n'est pas non plus possible d'envisager, à l'instar d'une approche strictement normative, qu'il ne peut y avoir qu'un "emboîtement strict et mécanique du sujet dans la norme» (Le Blanc, 2004, p. 172). Autrement dit, la construction du sujet est à envisager dans des rapports interpénétrés d'assujettissement des formés aux règles enseignées en formation mais aussi d'émancipation et non de subordination à ces dernières (Butler, 2005).

Sébastien Chaliès

Université de Toulouse 2, ESPE de Toulouse Midi-Pyrénées sebastien.chalies@univ-tlse2.fr

Cyrille Gaudin

Université de Limoges, ESPE de l'Académie de Limoges cyrille.gaudin@unilim.fr

Hervé Tribet

Université de Toulouse 3, Faculté des sciences du sport et du mouvement humain herve.tribet@univ-tlse3.fr

BERTONE S., CHALIÈS S. \& CLOT Y. (2009). «Contribution d'une théorie de l'action à la conceptualisation et à l'évaluation des pratiques réflexives dans des dispositifs de formation initiale des enseignants». Le Travail humain, $\mathrm{n}^{\circ} 72(2)$, p. 104-125.

BOHMAN J. (2011). «Réflexivité, agentivité et contrainte. Les paradoxes de la sociologie de la connaissance de Bourdieu ». In M. De Formel \& A. Ogien (dir.), Bourdieu : théoricien de la pratique. Paris : EHESS. p.21-47. 
BROPHY J. (2004). Using video in teacher education. Oxford: Elsevier.

BROUWER C. N. (2011). Equipping Teachers Visually. Zoetermeer : Kennisnet.

BURBULES N. C. (2008). «Tacit Teaching ». Educational Philosophy and Theory, $\mathrm{n}^{\circ} 40(5), \mathrm{p} .666-677$.

BUTLER J. (2005). Humain, inhumain : le travail critique des normes. Paris : La Découverte.

CALANDRA B. \& BRANTLEY-DIAS L. (2010). «Using digital video editing to shape novice teachers: A generative process for nurturing professional growth ». Educational Technology, n50(1), p. 13-17.

CASH M. (2009). «Normativity is the mother of intention: Wittgenstein, normative practices and neurological représentations ». New Ideas in Psychology, n²7, p. 133-147.

CAVELL S. (1996). Les voix de la raison. Paris : Éd. du Seuil.

CHALIÈS S., AMATHIEU J. \& BERTONE S. (2013). «Former les enseignants pour accroître leur satisfaction au travail : propositions théoriques et illustrations empiriques». Le Travail humain, $\mathrm{n}^{\circ}$ 76(3), p.309-334

CHALIÈS S., BERTONE S., FLAVIER E. \& DURAND M. (2008). «Effects of collaborative mentoring on the articulation of training and classroom situations: A case study in the French school system ". Teaching and Teacher Education, $\mathrm{n}^{\circ} 24(3)$, p. 550-563.

CHALIÈS S., BRUNO F., MÉARD J. \& BERTONE S. (2010). «Training preservice teachers rapidly: The need to articulate the training given by university supervisors and cooperating teachers». Teaching and Teacher Education, $\mathrm{n}^{\circ} 26$, p.764-774.

CHALIÈS S. \& RAYMOND J. (2008). «Conseiller sur le métier : une nécessaire reconstruction du métier». Revue EP.S., n०330, p.53-58.

CHAUVIRÉ C. (2003). Voir le visible: la seconde philosophie de Wittgenstein. Paris : PUF.

CHAUVIRÉ C. (2004). Le moment anthropologique de Wittgenstein. Paris : Kimé.

CHAUVIRÉ C. (2009). L'immanence de l'égo. Paris : PUF.

CHAUVIRÉ C. (2010). Wittgenstein en héritage. Philosophie de l'esprit, épistémologie, pragmatisme. Paris : Kimé.

CIAVALDINI-CARTAUT S. (2016). «Multimodalité numérique en situation de supervision de stage». Éducation permanente, $\mathrm{n}^{\circ} 206$, p.61-74.

CLOTY. (1999). La fonction psychologique du travail. Paris : PUF.

CLOT Y. (2008). Travail et pouvoir d'agir. Paris : PUF.

COMETTI J.-P. (2004). Ludwig Wittgenstein et la philosophie de la psychologie. Paris: PUF.

DAVIS A. (2009). «Examples as method? My attempts to understand assessment and fairness in the spirit of the later Wittgenstein ». Journal of Philosophy of Education, $\mathrm{n}^{\circ} 43$, p. 371-389.

DESCOMBES V. (2004). Le complément de sujet. Enquête sur le fait d'agir soi-même. Paris : Gallimard.
DEWEY J. (1990). Démocratie et éducation. Paris : Armand Colin.

DURAND M. (2008). «Un programme de recherche technologique en formation des adultes. Une approche enactive de l'activité humaine et l'accompagnement de son apprentissage/développement ». Éducation et didactique, $\mathrm{n}^{\circ} 2(2)$, p. $1-25$.

DURAND M. \& FILLIETAZ L. (2009). Travail et formation des adultes. Paris : PUF.

FLANDIN S. (2015a). Analyse de l'activité d'enseignants stagiaires du second degré en situation de vidéoformation autonome: contribution à un programme de recherche technologique en formation. Thèse de doctorat, sciences de l'éducation, université Blaise-Pascal.

FLANDIN S. (2015b). «Trois approches contrastées de la vidéoformation dans le paysage francophone. Tensions épistémologiques et effets en formation ». In L. Ria (dir.), Former les enseignants au xxle siècle. Volume 1 : Établissement formateur et vidéoformation. Bruxelles : De Boeck, p.151-159.

FLANDIN S. \& RIA L. (2014). «Un programme technologique basé sur l'analyse de l'activité réelle des enseignants débutant au travail et en vidéoformation». Activités, $\mathrm{n}^{\circ} 11(2)$, p. 172-187.

FLANDIN S., LEBLANC S. \& MULLER A. (2015). «Vidéoformation "orientée activité" : quelles utilisations pour quels effets sur les enseignants? ». In V. Lussi Borer, M. Durand \& F. Yvon (dir.), Analyse du travail et formation dans les métiers de l'éducation. Bruxelles : De Boeck, p.179-198.

GAUDIN C. \& CHALIÈS S. (2012). «L'utilisation de la vidéo dans la formation professionnelle des enseignants novices». Revue française de pédagogie, $\mathrm{n}^{\circ} 178, \mathrm{p} .115-130$.

GAUDIN C. \& CHALIÈS S. (2015). «Video use in teacher education and professional development: A literature review ». Educational Research Review, n ${ }^{0}$, p. 41-67.

GAUDIN C. \& CHALIÈS S. (2016). «A study of the influence of a video-based training program on preservice teachers' teaching activity ». In V. Lussi Borer, S. Flandin \& C. Gaudin (dir.), Understanding teachers' experiences in videoenhanced education: Implications for the design of instructional programs. Communication au colloque EARLISIG 11: "Diversity and Inclusion as a Challenge for Teacher Education », organisé à Zurich (Suisse) du 20 au 22 juin 2016.

GAUDIN C., FLANDIN S., RIA L. \& CHALIÈS S. (2014). «An exploratory study of the influence of video viewing on preservice teachers' teaching activity: Normative versus developmental approaches». Form@re, n²(14), p.21-50.

GAUDIN C., PERROT F., CHALIÈS S., ESCALIÉ G. \& RAYMOND J. (2014). «La visite de stage : de l'observation au conseil à partir des règles de métier ». Revue EP.S., n³53, p.38-41.

GRAMMONT. F., LEGRAND D. \& LIVET P. (2011). Naturalizing intention in action. Cambridge : MIT Press.

KOC M. (2011). « Let's make a movie: Investigating pre-service teachers' reflections on using video-recorded role playing cases in Turkey ». Teaching and Teacher Education, $\mathrm{n}^{\circ} 27(2)$, p. 95-106. 
LÄHTEENMÄKI M. (2003). "On rules and rule following: Obeying rules blindly». Language and Communication, $n^{\circ} 23(1)$, p. 45-61.

LASAGABASTER D. \& SIERRA J.-M. (2011). «Classroom observation: Desirable conditions established by teachers ». European Journal of Teacher Education, n³4(4), p. 449-463.

LAUGIER S. (2009). Wittgenstein : les sens de l'usage. Paris: Vrin.

LAUGIER S. \& CHAUVIRÉ C. (2006). Lire les recherches philosophiques de Wittgenstein. Paris : Vrin.

LAVE J. \& WENGER E. (1991). Situated learning: Legitimate peripheral participation. Cambridge (Royaume-Uni) : Cambridge University Press.

LE BLANC G. (1998). Canguilhem et les normes. Paris : PUF.

LE BLANC G. (2004). Les maladies de l'homme normal. Bègles: Éditions du Passant.

LEBLANC S. (2012). Conception d'environnements vidéo numériques de formation. Développement d'un programme de recherche technologique centré sur l'activité dans le domaine de l'éducation. Note de synthèse pour l'Habilitation à diriger les recherches, sciences de l'éducation, université Paul-Valéry-Montpellier 3.

LEBLANC S. (2014). «Expériences mimétiques en vidéoformation et transformations de l'activité professionnelle». Recherche et formation, $\mathrm{n}^{\circ} 75, \mathrm{p} .37-50$.

LEBLANC S. \& VEYRUNES P. (2011). «"Vidéoscopie" et modélisation de l'activité enseignante ». Recherche et formation, $\mathrm{n}^{\circ} 68, \mathrm{p} .139-152$.

LEGRAND D. (2007). «Pre-relective self-as-subject from experiential and empirical perspectives». Consciousness and Cognition, n 16, p. 583-599.

LIVET P. (1993). «Théorie de l'action et conventions». In P. Ladrière, P. Pharo \& L. Quéré (dir.). La théorie de l'action. Le sujet pratique en débat. Paris : CNRS Éd., p.291-319.

LUSSI BORER V. \& MULLER A. (2014a). «Exploiter le potentiel des processus de renormalisation en formation à l'enseignement». @ctivités, n ${ }^{11}(2)$, p. 129-142.

LUSSI BORER V. \& MULLER A. (2014b). «Connaître l'activité des enseignants en formation sur la plateforme Néopass @ction ». Recherche et formation, n 75, p. 65-80.

LUSSI BORER V. \& RIA L. (2015). «Concevoir et expérimenter un laboratoire d'analyse vidéo de l'activité enseignante au sein d'un établissement scolaire». In V. Lussi Borer, M. Durand \& F. Yvon (dir), Analyse du travail et formation dans les métiers de l'éducation. Bruxelles : De Boeck, p.219-238.

LUSSI BORER V., RIA L., DURAND M. \& MULLER A. (2014). "How do teachers appropriate learning objects through critical experiences? A study of a pilot in-school collaborative video learning lab». Form@re, n`14(2), p.63-74.

MARSH B., MITCHELL N. \& ADAMCZYK P. (2009). «Interactive video technology: Enhancing professional learning in initial teacher education ». Computers and Education, n54(3), p.742-748.

MASATS D. \& DOOLY M. (2011). « Rethinking the use of video in teacher education: A holistic approach». Teaching and Teacher Education, n²7(7), p.1151-1162.
MERSETH K. K. (1994). «Instructional methods and conceptual orientations in the design of teacher education programs: The example of simulations, hypermedia, and cases». In K. R. Howey (dir.), The professional development of teacher educators. Cincinnati : Ablex Publishing, p.139-174.

MOUSSAY S. (2013). «Conception d'un dispositif de vidéoformation centré sur le travail réel et professionnalisation au métier d'enseignant en STAPS ». eJRIEPS, n²9, p. 4-26.

NARBOUX J.-P. (2003). «Incommensurabilité et exemplarité : aliénation logique et problème des universaux». Archives de Philosophie, n66, p. 437-447.

NELSON K. (2008). «Wittgenstein and contemporary theories of word Learning ». New Ideas in Psychology, n 4, p. 1-13.

OGIEN A. (2007). Les formes sociales de la pensée. La sociologie après Wittgenstein. Paris : Armand Colin.

OSTROSKY M. M, MOUZOUROU C., DANNER N. \& ZAGHLAWAN H. Y. (2013). «Improving teacher practices using microteaching: Planful video recording and constructive feedback». Young Exceptional Children, $\mathrm{n}^{\circ} 16(1)$, p. 16-29.

PUVIRAJAH A. \& CALANDRA B. (2015). «Embodied experiences in virtual worlds role-play as a conduit for novice teacher identity exploration: A case study». Identity: An International Journal of Theory and Research, $\mathrm{n}^{\circ} 15(1)$, p. 23-47.

RECOULES N. \& CHALIÈS S. (2011). «Apprendre à faire apprendre». Revue EP.S., n`349, p. 34-37.

RECOULES N., ESCALIÉ G., GAUDIN C. \& CHALIÈS S. (2012). "Apprendre à faire apprendre : illustrations d'une démarche». Revue EP.S., n³53, p.3-7.

ROCHE L. \& GAL-PETITFAUX N. (2015). «A video-enhanced teacher learning environment based on multimodal resources: A case study in PETE». Journal of e-Learning and Knowledge Society, $\mathrm{n}^{\circ} 11(2)$, p. 91-110.

ROGOFF B., MATUSOF E. \& WHITE C. (1996). « Models of teaching and learning: participation in a community of learners ». In D. R. Olson \& N. Torrance (dir.), The handbook of education and human development: New models of learning, teaching and schooling. Oxford: Basil Blackwell Publishers, p. 388-414.

SANTAGATA R. (2014). «Towards ambitious teaching: Using video to support future teachers reasoning about evidence of student learning ». Recherche et formation, $\mathrm{n}^{\circ} 75$, p. 95-110.

SANTAGATA R. \& GUARINO J. (2011). "Using Video to Teach Future Teachers to Learn from Teaching ». ZDM The International Journal of Mathematics Education, $\mathrm{n}^{\circ}$ 43(1), p. 133-145.

SANTAGATA R. \& YEH C. (2014). «Learning to teach mathematics and to analyze teaching effectiveness: Evidence from a video-and practice-based approach». Journal of Mathematics Teacher Education, ${ }^{\circ} 17(6)$, p. 491-514.

SCHWARTZ, Y. (2007). «Un bref aperçu de I'histoire du concept culturel d'activité». Activités, n 4(2), p. 122-133.

SEARLE J. R. (1998). La construction de la réalité sociale. Paris : Gallimard. 
SHERIN M. (2004). «New perspectives on the role of video in teacher education ». In J. Brophy (dir.), Using video in teacher education. Oxford: Elsevier, p. 1-28.

SHERIN M. G. \& van ES E. A. (2005). «Using video to support teachers' ability to notice classroom interactions». Journal of Technology and Teacher Education, n' 13(3), p. 475-491.

SIMONDON G. (2008). Imagination et invention. Lonrai : La Transparence.

SYRING M., KLEINKNECHT M., BOHL T., KUNTZE S., REHM M.-L. M. \& SCHNEIDER J.-M. A. (2015). «How problembased or direct instructional case-based learning environments influence secondary school pre-service teachers' cognitive load, motivation and emotions: A quasiexperimental intervention study in teacher education». Journal of Education and Human Development, $n^{\circ} 4(4)$, p. 115-129.

TARDIF J., BORGES C. \& MALO A. (2013). Le virage réflexif en éducation. Bruxelles : De Boeck.

TAYLOR C. (1995). "Suivre une règle». Critique, n579-580, p.555-572.

THEUREAU J. (2004). Le cours d'action: méthode élémentaire. Toulouse : Octarès.

THEUREAU J. (2006). Le cours d'action : méthode développée. Toulouse : Octarès.
TRIPP T. \& RICH P. (2012). « Using video to analyze one's own teaching». British Journal of Educational Technology, $\mathrm{n}^{\circ}$ 43(4), p. 678-704.

VARELA F.-J. (1989). Autonomie et connaissance. Essai sur le vivant. Paris : Éd. du Seuil.

VYGOTSKI L. (1985). «Le problème de l'enseignement et du développement mental à l'âge scolaire». In B. Schneuwly \& J.-P. Bronckart (dir.), Vygotsky aujourd'hui. Lausanne : Delachaux et Niestlé, p. 95-118.

VYGOTSKI L. (1997). Pensée et langage. Paris : La Dispute.

WILLIAMS M. (2002). «Tout est-il interprétation?». In C. Chauviré \& A. Ogien (dir.), La régularité. Paris : EHESS, p.207-233.

WINCH P. (2009). L'idée d'une science sociale et sa relation à la philosophie. Paris : Gallimard.

WITTGENSTEIN L. (2004). Recherches philosophiques. Paris : Gallimard.

YUNG B., WONG S., CHENG M., HUI C. \& HODSON D. (2007). «Tracking preservice teachers' changing conceptions of good science teaching: The role of progressive reflection with the same video». Research in Science Education, n³7(3), p. 239-259. 
